\title{
Treatment Patterns and Outcomes Among Elderly Glioblastoma Patients in Riyadh, Saudi Arabia
}

\author{
Amal Marie ${ }^{1,2}$, Ahmed Maklad (1D ${ }^{1,3}$, Abdullah AlTwairgi ${ }^{4}$, Moemen Aly $\mathbb{D}^{1,5}$, Ashraf Elyamany $\mathbb{D}^{6}$, \\ Wafaa AlShaqweer ${ }^{7}$, Mohamed Senosy', Ali Balbaid' \\ 'Department of Radiation Oncology, Comprehensive Cancer Centre, King Fahad Medical City, Riyadh, Saudi Arabia; ${ }^{2}$ Clinical Oncology Department, \\ Ain Shams University, Cairo, Egypt; ${ }^{3}$ Clinical Oncology Department, Sohag University, Sohag, Egypt; ${ }^{4}$ Department of Medical Oncology, King Fahad \\ Medical City, Riyadh, Saudi Arabia; ${ }^{5}$ Department of Radiotherapy and Nuclear Medicine, South Egypt Cancer Institute, Assiut University, Assiut, Egypt; \\ ${ }^{6}$ Medical Oncology Department, SECl, Assiut University, Egypt/KSMC, Riyadh, Saudi Arabia; ${ }^{7}$ Pathology Department, King Fahad Medical City, Riyadh, \\ Saudi Arabia
}

Correspondence: Ashraf Elyamany, Email elyamany23@gmail.com

Background: Management of elderly patients with glioblastoma (GBM) is a controversial scenario and needs careful assessment and selection for aggressive radical treatment and chemotherapy protocols vs short-course radiotherapy without chemotherapy.

Methods: We evaluated treatment patterns and outcome among elderly GBM patients treated in KFMC, Riyadh. The primary endpoint is overall survival (OS) and the secondary endpoint is progression-free survival (PFS); patients were reviewed regarding radiotherapy (Rth) fractionation modalities, surgery, and chemotherapy (CTR) given in correlation to PFS, OS.

Results: Fifty-nine patients were recruited in our study with median age 66 (range: 60-81) years, and 47 (80\%) were males. Thirtyseven patients $(62.7 \%)$ had ECOG performance status (PS) $\geq 2$, and 22 patients $(37.3 \%)$ had PS $<2$. Gross total resection (GTR) and subtotal resection (STR) were done in $49(82.9 \%)$ patients, and the median follow-up was 12 months. Thirty-eight (64\%) patients received conventional Rth 60 Gray (Gy)/30 fractions or equal doses and 21 (36\%) patients received hypofractionation Rth (40 Gy/15, $25 \mathrm{~Gy} / 5$ or $30 \mathrm{~Gy} / 10$ fractions). The median OS was 12 months (95\%CI: 9.52-14.48). Receiving conventional Rth and completion of six months adjuvant CTR were significant factors for O.S ( $P=0.043$ and 0.026$)$, respectively. The median PFS was nine months $(95 \%$ CI: 6.13-11.87). For univariate analysis, PS, time to start adjuvant treatment, and completion of six months CTR were significant factors for PFS.

Conclusion: Conventional Rth and completion of adjuvant CTR lead to better OS, while earlier start of adjuvant treatment and the completion of adjuvant CTR were associated with a better PFS.

Keywords: glioblastoma, elderly, chemotherapy, radiotherapy

\section{Introduction}

Glioblastoma (GBM) is the most common malignant primary brain tumor among adults. ${ }^{1}$ The reported median age at diagnosis is about 65 years old, with a rapidly increasing incidence among patients aged more than 65 years, which almost doubled from 5.1 per 100,000 in 1970 to 10.6 per 100,000 in $1990 .^{2}$ The standard treatment for newly diagnosed GBM with good performance status and age less than 70 years is the addition of temozolomide to radiotherapy followed by six cycles of adjuvant temozolomide as per the Phase III study done by Stupp et al, 2005, median patients age was 56 years, and $84 \%$ of patients had undergone debulking surgery. At a median follow-up of 28 months, the median survival was 14.6 months with radiotherapy plus temozolomide and 12.1 months with radiotherapy alone. ${ }^{3}$ The median survival among all GBM patients ranges from 12 to 15 months from diagnosis despite aggressive treatment, while it was markedly shorter (only four to five months) among elderly patients. ${ }^{4,5}$ It is evident from existing literature that the elderly GBM patients usually receive less effective therapies including surgery, radiotherapy (Rth), and chemotherapy (CTR) compared to their younger counterparts. ${ }^{6,7}$ There is even a clearly defined survival benefit for elderly GBM patients receiving longer-course radiotherapy compared to best supportive care. ${ }^{8}$ In contrast, Roa et al explained a 
noninferiority of hypofractionated Rth course of 40 Gray (Gy) compared to standard Rth of 60 Gy among patients aged 60 years and above with minimum ECOG PS $3 .^{9}$

In this study, we reviewed the different treatment options offered to elderly patients with GBM at a tertiary care center in Riyadh and the impact on overall survival (OS) and progression-free survival (PFS).

\section{Materials and Methods}

\section{Study Design}

A retrospective cohort study was carried out in the Department of Radiation Oncology, King Fahad Medical City (KFMC), Riyadh, Saudi Arabia. The study was approved by the local Ethics Committee of KFMC. Data were obtained from the electronic medical records of the hospital for elderly GBM cases.

\section{Eligibility Criteria}

The study included all histologically proven, newly diagnosed elderly (age $\geq 60$ years) GBM cases, who were treated at our institute between January 2008 and January 2018. The study excluded patients with recurrent disease at initial presentation.

\section{Treatment Modalities}

All patients underwent surgical intervention either by biopsy only, subtotal resection (STR), or gross total resection (GTR), followed by adjuvant treatment except for one patient who died before starting adjuvant treatment. Adjuvant treatment included either Rth only or Rth concurrent with temozolomide. Radiotherapy included either conventional fractionation (59.4 Gy/33 fractions or $60 \mathrm{~Gy} / 30$ fractions) or hypofractionation (30 Gy/10 fractions or $40 \mathrm{~Gy} / 15$ fractions). A proportion of patients with an Eastern Cooperative Oncology Group (ECOG) performance status (PS) $\leq 2$ were offered adjuvant CTR after completion of radiation.

\section{Definition of Survival}

OS was defined as the interval between initial surgery or pathology diagnosis and date of death (where applicable). In patients still alive at the end of the study period, data were censored on January 1, 2018. PFS was defined as the interval between initial surgery or pathology diagnosis and the date of first progression or recurrence or death (if no progression was reported) or until the last evaluation date.

\section{Data Collection}

The data were collected from electronic medical records through a data collection form which was developed to collect patient characteristics, pathology, external beam radiotherapy (EBRT) and CTR details, and progression and survival times.

\section{Statistical Analysis}

OS and PFS are calculated by using the Kaplan-Meier method, and the difference in survival curves was compared by using the $\log$ rank test. Different categorical variables are compared with the chi-squared test. The level of significance was set at $P<0.05$ and $P$-values are based on two-sided tests. Multivariate analysis using the Cox proportional hazards model is performed to define various potential prognostic factors. All analyses were performed using the SPSS version (IBM SPSS, Armonk, NY, USA).

\section{Compliance with Ethical Standards}

This study was approved by Ethical Committee and get IRB approval No. 18-201 April 2018. It was conducted in accordance with the Declaration of Helsinki of 1975. The study did not interfere with patient management and was done retrospectively, collected using medical records without any violation of patients' confidentiality so a consent from the 
patient was not needed. Data will be available if needed to be reviewed by journal reviewers after approval of the research center King Fahad Medical City (KFMC).

\section{Results}

A total of 59 patients met the inclusion criteria out of 158 newly diagnosed GBM cases who were treated in our institution between January 2008 and January 2018. One case died before adjuvant treatment. The median age at diagnosis was 66 years (ranging between 60 and 81 years); 47 patients were males (79.7\%) and 12 were females $(20.3 \%)$. Forty-nine patients $(83 \%)$ were diagnosed with GBM and 10 patients $(16.9 \%)$ with GBM variants. Fifty-three $(89.8 \%)$ patients had a unilateral tumor and six (10.2\%) patients had bilateral disease. Patients' demographic features and clinical characteristics are included in Table 1, tumor locations were summarized in Table 2.

Patients with ECOG PS $\geq 2$ were 37 patients $(62.7 \%)$ and 22 patients $(37.3 \%)$ had PS $<2$. All patients underwent surgery with 10 (16.9\%) patients had biopsy only, 42 patients had STR (71.2\%) and seven patients (11.9\%) had GTR. The median time to start adjuvant treatment was 1.58 months (0.43-6.54 months). Fifty-eight patients received adjuvant treatment; 22 patients (37.3\%) received Rth only, 13 patients (22\%) received concurrent chemoradiotherapy and 23 patients (39\%) received concurrent chemoradiotherapy followed by adjuvant CTR. Among patients received adjuvant CTR; 11 patients (47.8\%) received $\geq 6$ cycles and 12 patients (52.2\%) received $<6$ cycles. Regarding Rth fractionation; 38 patients $(64.4 \%)$ received conventional fractionation $(59.4 \mathrm{~Gy} / 33$ fractions or $60 \mathrm{~Gy} / 30$ fractions) and 21 patients (35.6\%) received hypofractionation (25 Gy/5, $30 \mathrm{~Gy} / 10$ fractions or $40 \mathrm{~Gy} / 15$ fractions).

Table I Patients' Demographic Features and Clinical Characteristics

\begin{tabular}{|c|c|}
\hline Variables & $\mathbf{N}(\%)$ \\
\hline Mean age (years) & $66(60-81)$ \\
\hline \multicolumn{2}{|l|}{ KPS group } \\
\hline$<2$ & $22(37.3 \%)$ \\
\hline$\geq 2$ & $37(62.7 \%)$ \\
\hline \multicolumn{2}{|l|}{ Gender } \\
\hline Male & 47 (79.7\%) \\
\hline Female & $12(20.3 \%)$ \\
\hline \multicolumn{2}{|l|}{ Surgery type: } \\
\hline Biopsy & $10(16.9 \%)$ \\
\hline STR & 42 (7I.2\%) \\
\hline GTR & 7 (II.9\%) \\
\hline \multicolumn{2}{|l|}{ Adjuvant treatment types } \\
\hline Radiotherapy & $22(37.3 \%)$ \\
\hline CCRT & $13(22 \%)$ \\
\hline CCRT + Adj CTR & $23(39 \%)$ \\
\hline No treatment & I (I.7\%) \\
\hline \multicolumn{2}{|l|}{ Start period of adjuvant: } \\
\hline$<2 \mathrm{~ms}$ & $42(72.4 \%)$ \\
\hline$\geq 2 \mathrm{~ms}$ & $16(27.6 \%)$ \\
\hline \multicolumn{2}{|l|}{ Radiotherapy fractionation } \\
\hline Hypofractionation & $20(33.9 \%)$ \\
\hline Conventional & $38(66.1 \%)$ \\
\hline \multicolumn{2}{|l|}{ No. of cycles of adjuvant CTR: } \\
\hline$\geq 6$ & $12(52.2 \%)$ \\
\hline$<6$ & II (47.8\%) \\
\hline
\end{tabular}


Table 2 Tumor Locations

\begin{tabular}{|c|c|c|}
\hline \multicolumn{2}{|c|}{ Tumor Location } & $\mathbf{N}(\%)$ \\
\hline \multicolumn{2}{|l|}{ Frontal } & $17(28.8)$ \\
\hline \multicolumn{2}{|l|}{ Parietal } & $4(6.8)$ \\
\hline \multicolumn{2}{|l|}{ Temporal } & $13(22)$ \\
\hline \multicolumn{2}{|l|}{ Occipital } & I (I.7) \\
\hline \multicolumn{2}{|l|}{ Multifocal } & 7 (II.9) \\
\hline \multicolumn{2}{|l|}{ Others } & I (I.7) \\
\hline \multicolumn{2}{|c|}{ More than one adjacent lobe } & $16(27.1)$ \\
\hline \multirow[t]{3}{*}{ Hemisphere } & Bilateral & $6(10.1)$ \\
\hline & Left & $24(40.7)$ \\
\hline & Right & $29(49.2)$ \\
\hline
\end{tabular}

The median OS was 12 months (95\%CI; 9.52-14.48) Figure 1. For univariate analysis, receiving a conventional Rth and completion of six months adjuvant CTR were significant factors for O.S ( $P=0.043$ and 0.026 , respectively). For multivariate those were also significant $(P=0.035$ and 0.002 , respectively) Table 3 and Figures 2 and 3.

The median PFS was nine months (95\%CI: 6.13-11.87) Figure 4. For univariate analysis PS, time to start adjuvant treatment, and completion of six months CTR were significant factors for PFS. For multivariate analysis starting adjuvant treatment within two months and completed chemotherapy six months were significant factors $(P=0.032$ and 0.04 , respectively) Table 4 and Figures 5 and 6.

\section{Discussion}

There is no well-established standard of care for the treatment of glioblastoma in the elderly, which make decision for treatment of the fit elderly difficult. In the current study we aimed at reviewing the different treatment options offered to

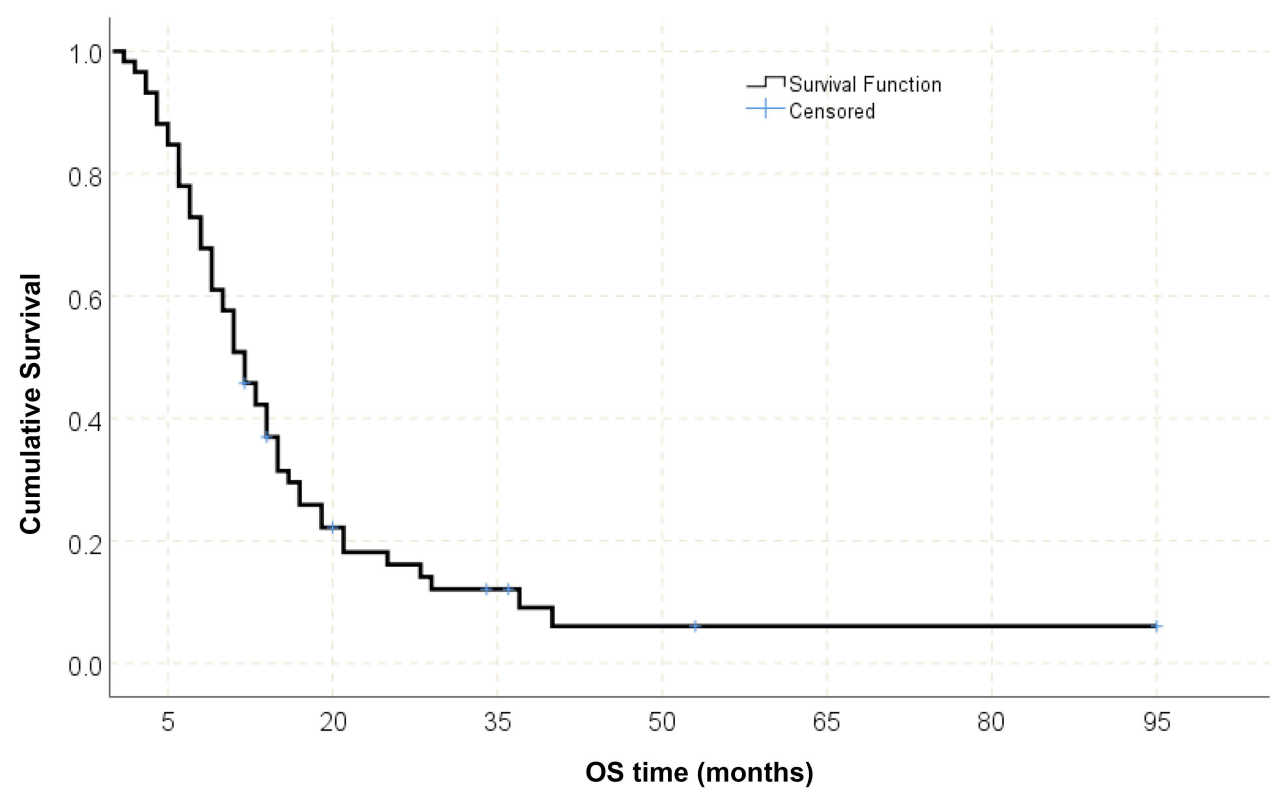

Figure I OS of all study groups of elderly GBM. 


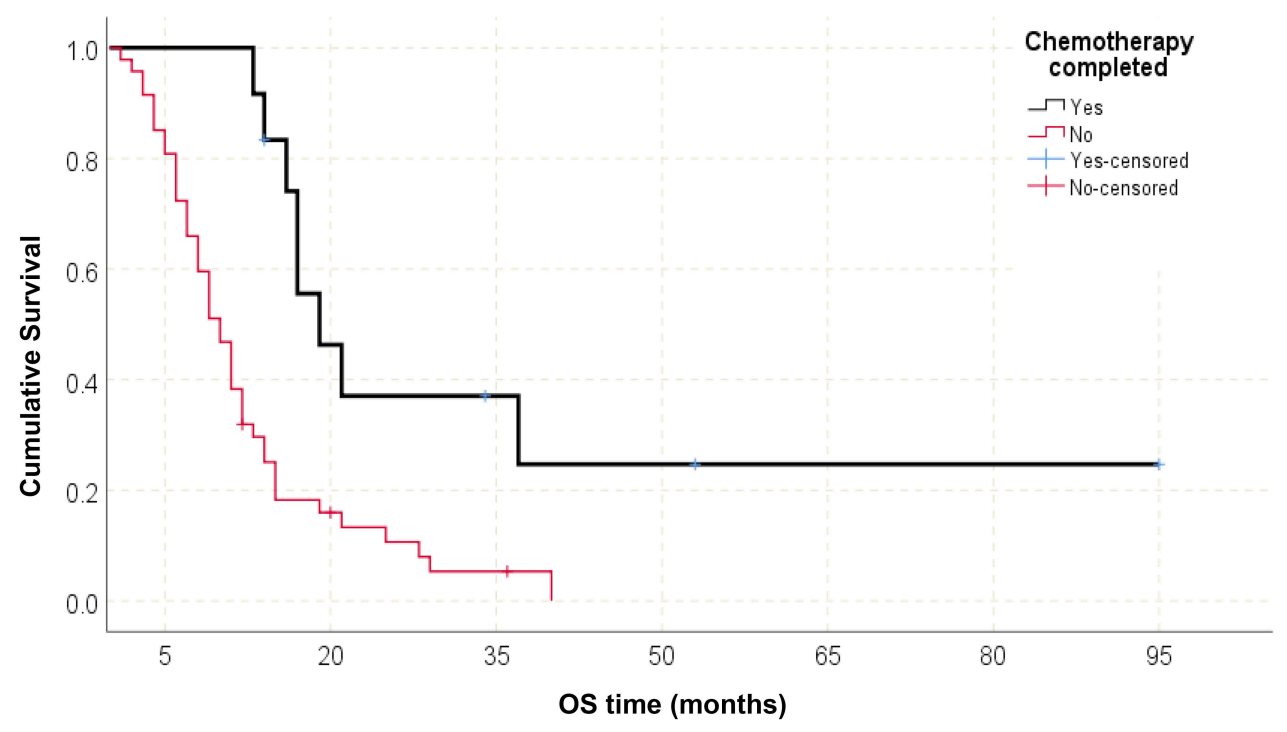

Figure 2 Effect of completed CTR on OS of elderly GBM patients.

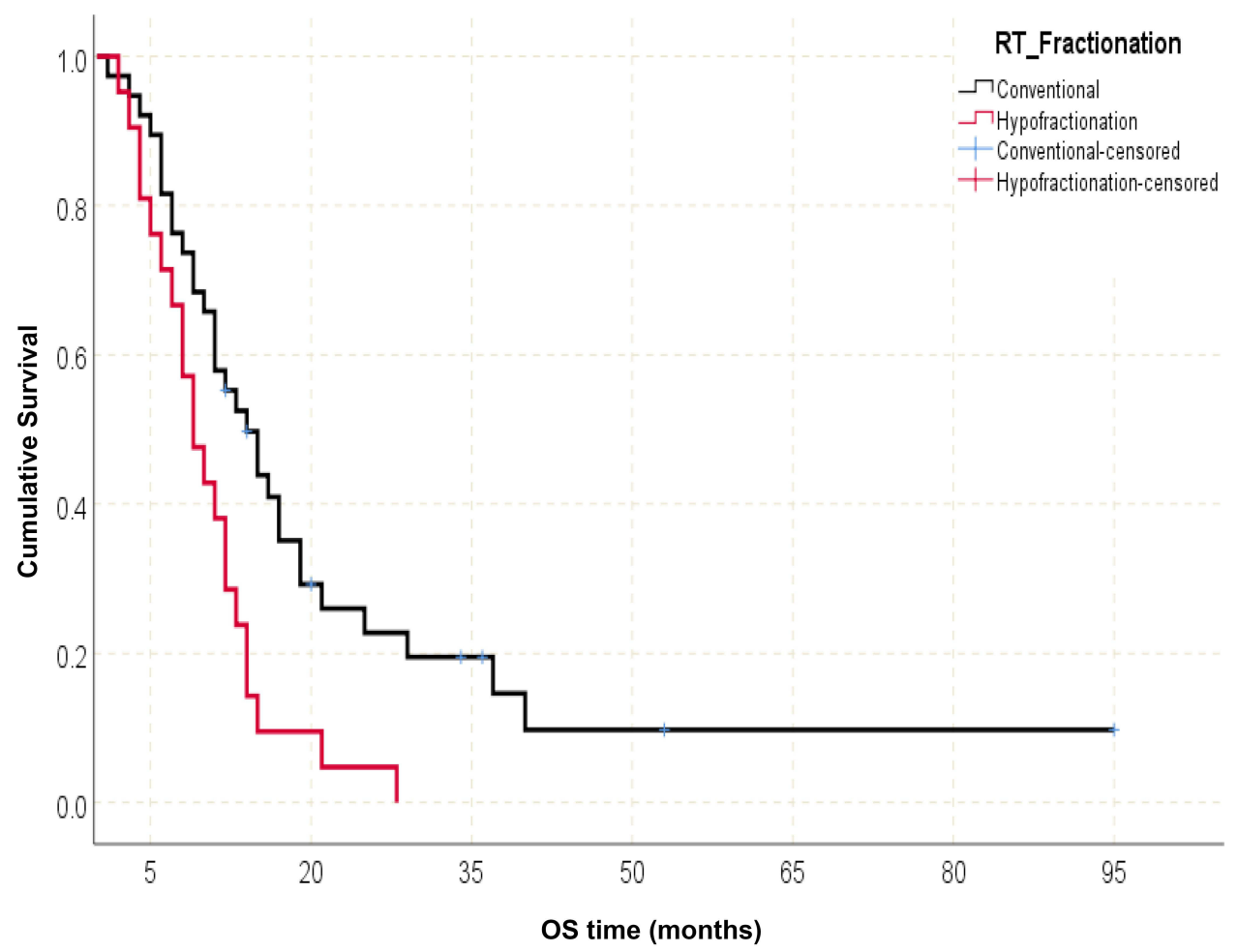

Figure 3 Effect of radiotherapy fractionation on OS of elderly GBM patients.

elderly patients with GBM at KFMC, and its impact on overall survival (OS) and progression-free survival (PFS). There is controversy about definition of elderly population. We decided to follow the Nordic trial inclusion criteria which included patients with age more than 60 years as this is more aligned with life expectancy in our population. ${ }^{10}$ The famous studies for elderly population like Wick et al., (NOA-08 study) did not include patients with concurrent plus adjuvant temozolomide, unlike Perry et al, study which added temozolomide to short-course radiation only, while our patients received more different treatment options according to treatment guidelines, in our study we tried to evaluate the benefit of different treatment modalities. ${ }^{11,12}$ The (OS) of our patients was 12 months which was comparable with 9.2 
Table 3 Analysis of Different Factors Affecting OS for Our Study Group of Elderly GBM

\begin{tabular}{|l|l|l|l|l|l|l|}
\hline & \multicolumn{2}{l}{ Univariate } & \multicolumn{2}{l|}{ Multivariate } \\
\cline { 2 - 7 } & Crude OR & $\mathbf{9 5 \% C l}$ (LL-UL) & P-value & Adjusted OR & (LL-UL) & P-value \\
\hline Age $(\geq 65$ years) & 1.69 & $0.3-9.56$ & 0.694 & 1.08 & $0.58-2.01$ & 0.819 \\
\hline ECOG $(0-1)$ & 1.30 & $0.26-6.45$ & 1 & 1.15 & $0.61-2.18$ & 0.658 \\
\hline Rth fractionation & 47.42 & $0.09-24977.75$ & 0.043 & 2.87 & $1.08-7.61$ & 0.035 \\
\hline Surgery type & 16.67 & $0.03-8897.91$ & 0.59 & 0.51 & $0.24-1.08$ & 0.08 \\
\hline Start adjuvant (<2 months) & 1.01 & $0.18-5.81$ & 1 & 0.90 & $0.42-1.9$ & 0.775 \\
\hline Concurrent CTR & 4.40 & $0.49-39.21$ & 0.229 & 0.55 & $0.19-1.58$ & 0.269 \\
\hline CTR completed & 7.33 & $1.37-39.18$ & 0.026 & 4.04 & $1.66-9.82$ & 0.002 \\
\hline
\end{tabular}

months for 1059 patients in the Scoccianti et al, study. ${ }^{13}$ The median PFS was nine months for our cases, and this was higher than the Ewelt study which showed 5.9 months PFS for 1201 patients. ${ }^{14}$ In a retrospective analysis of American patients more than 65 years of age with a new diagnosis of GBM between 1997 and 2009, median survival ranged between two months (for patients who received no postoperative therapy) and 11 months (for those who received standard combined chemoradiation). ${ }^{15}$ In almost all reported series in the literature, we found young age, good performance status, and safe optimal resection to be the well-known good prognostic factors in patients with GBM. ${ }^{16-}$ ${ }^{18}$ In the present study, we found conventional Rth and completion of six months adjuvant CTR were independent prognostic factors for overall survival. In our study extent of surgical resection was found to have no significant impact on either PFS or OS, which is not similar to most published data, may be related to a small proportion of patients that could achieve GTR (seven patients $11.9 \%$ underwent GTR). The results of meta-analysis including 34 studies showed

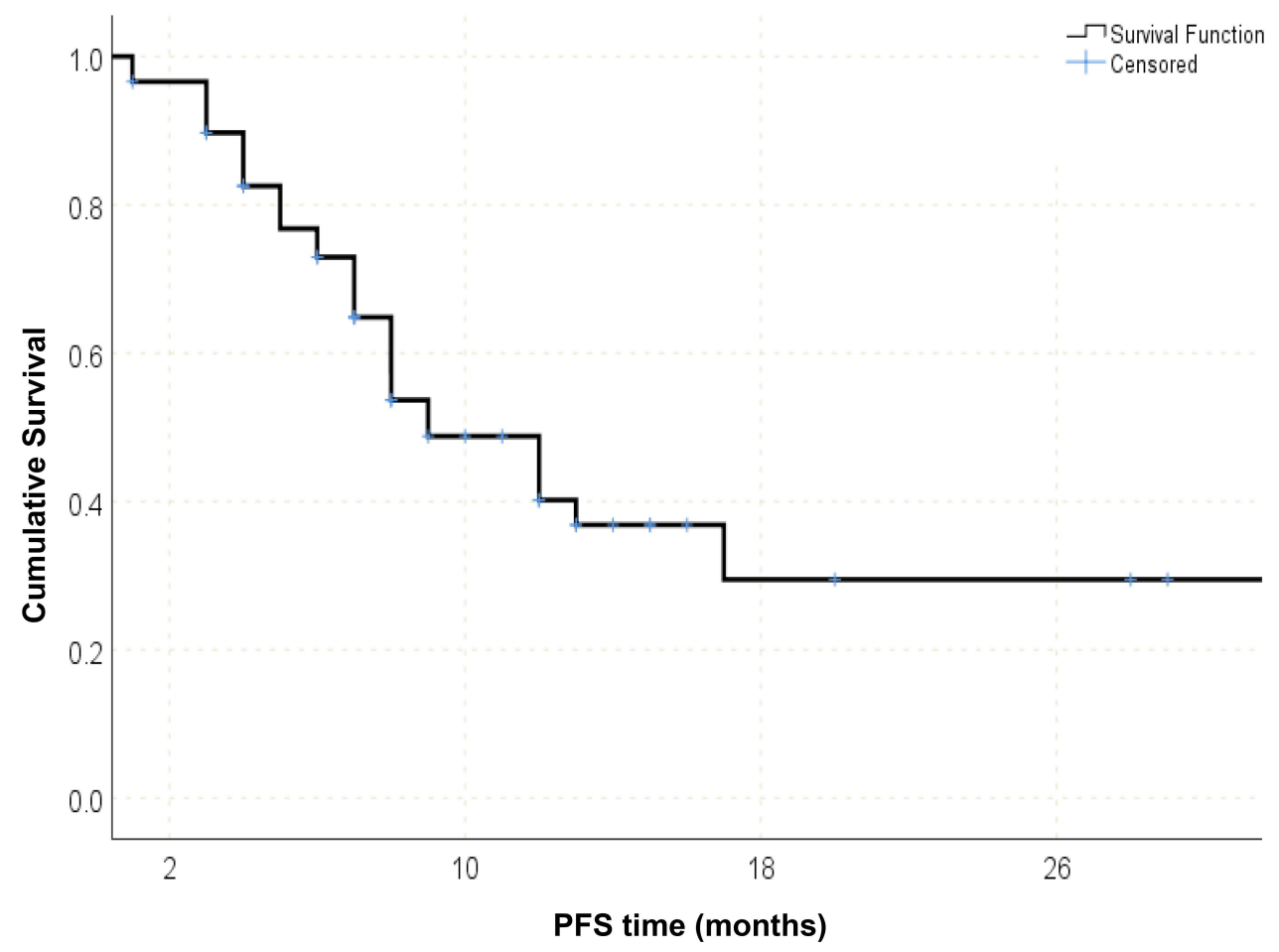

Figure 4 PFS of all study groups of elderly GBM. 


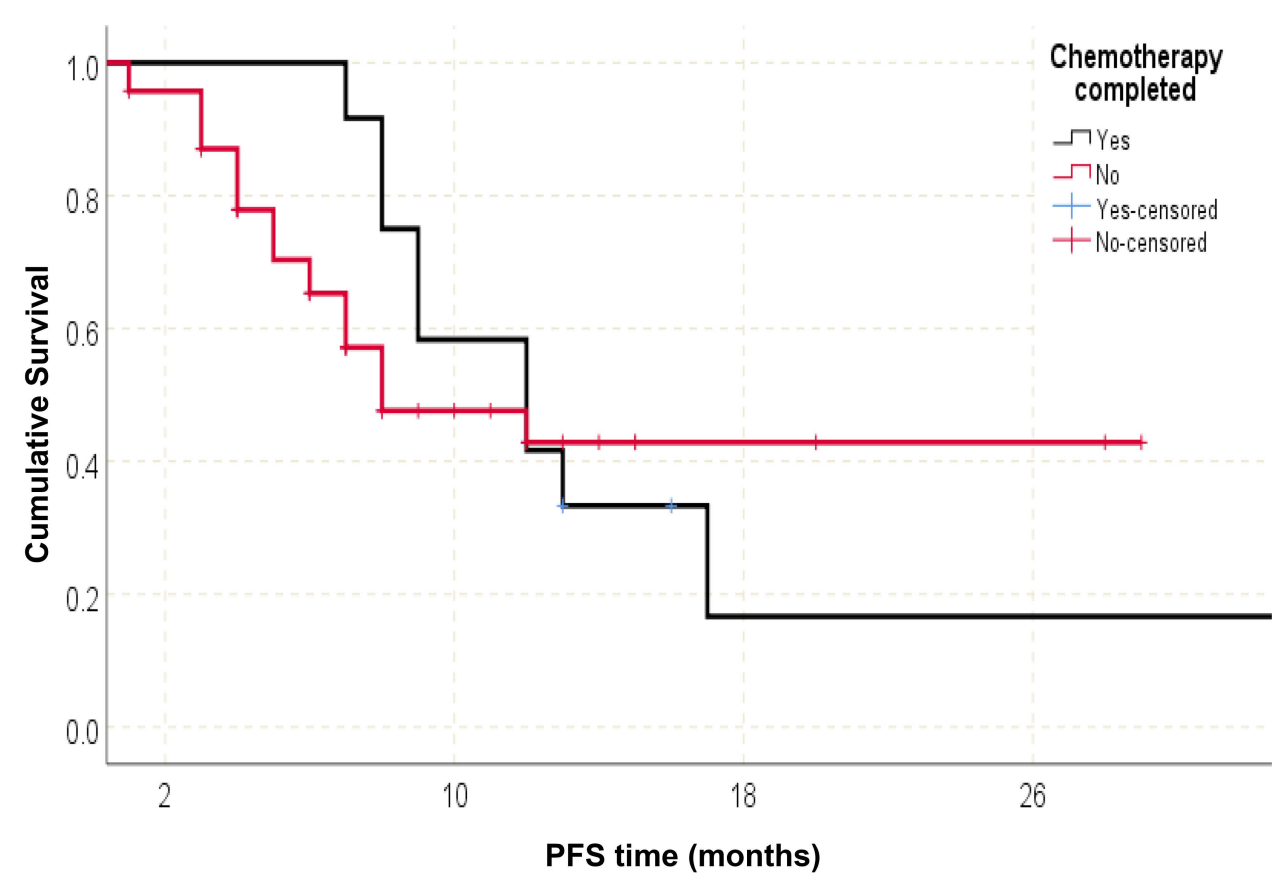

Figure 5 Effect of completed CTR on PFS of elderly GBM patients.

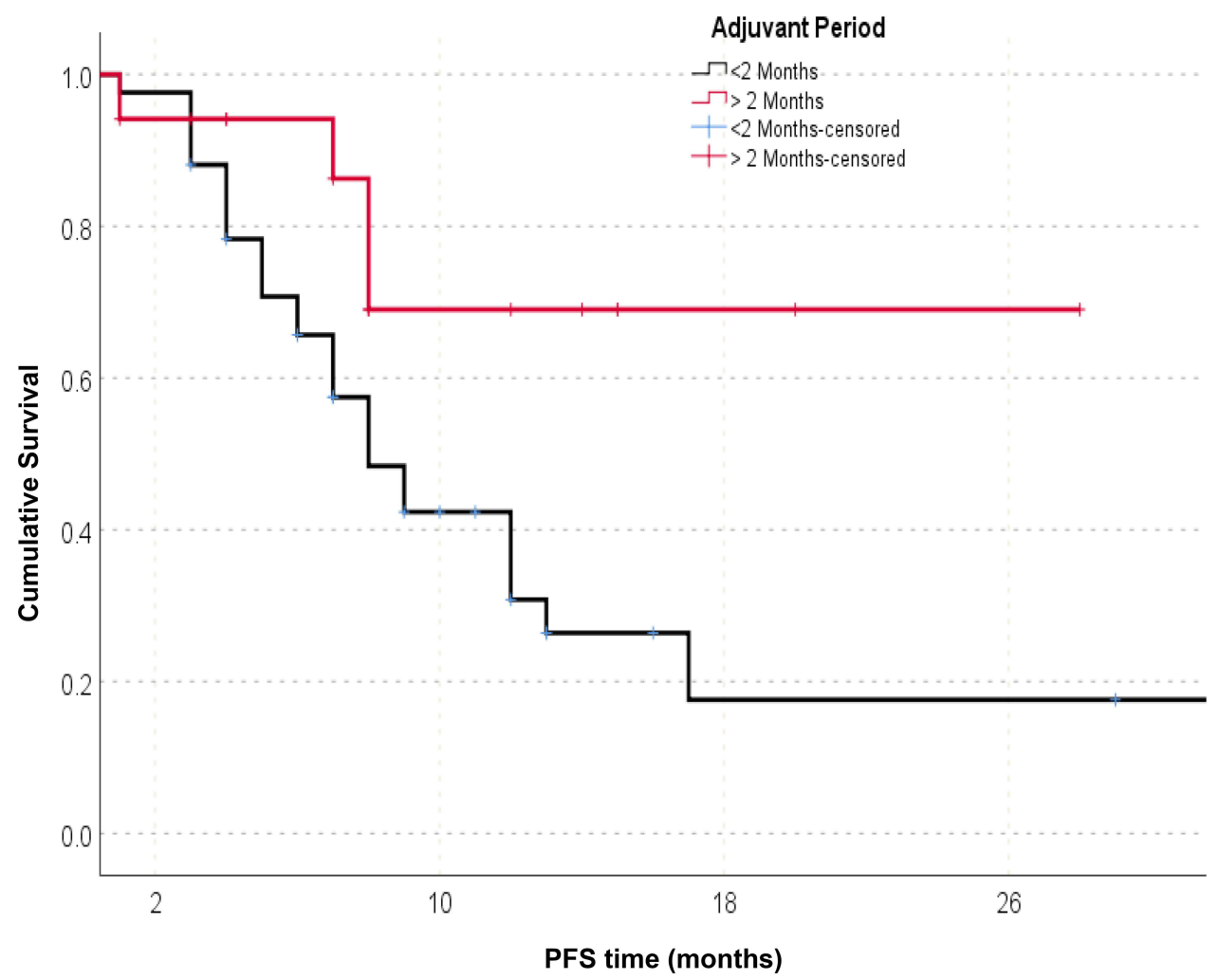

Figure 6 Effect of period to start adjuvant treatment on PFS of elderly GBM patients.

that surgical resection was superior to biopsy regarding OS (mean difference 3.88 months, 95\%CI: 2.14-5.62, $P<0.001) .{ }^{19}$ In a prospective randomized study conducted by Vuorinen et al discussed the extent of resection in elderly patients with malignant GBM aged $>65$ years found that surgical removal of the tumor prolonged survival 2.8 times than 
Table 4 Analysis of Different Factors Affecting PFS for Our Study Group of Elderly GBM

\begin{tabular}{|l|l|l|l|l|l|l|}
\hline & \multicolumn{2}{l}{ Univariate } & \multicolumn{2}{l|}{ Multivariate } \\
\cline { 2 - 7 } & Crude OR & $\mathbf{9 5 \% C l}$ (LL-UL) & P-value & Adjusted OR & (LL-UL) & P-value \\
\hline Age ( $\geq 65$ years) & 1.16 & $0.4 I-3.33$ & 0.778 & 1.12 & $0.5 I-2.46$ & 0.776 \\
\hline ECOG PS [0-I] & 0.20 & $0.06-0.66$ & 0.006 & 1.90 & $0.85-4.27$ & 0.117 \\
\hline Rth fractionation & 0.49 & $0.17-1.44$ & 0.192 & 1.57 & $0.41-6.07$ & 0.510 \\
\hline Surgery type & 1.33 & $0.33-5.3$ & 0.741 & 0.55 & $0.21-1.45$ & 0.223 \\
\hline Start adjuvant (<2 months) & 0.15 & $0.04-0.56$ & 0.003 & 3.50 & $1.12-10.96$ & 0.032 \\
\hline Concurrent CTR & 0.49 & $0.17-1.42$ & 0.185 & 1.80 & $0.43-7.53$ & 0.421 \\
\hline CTR completed & 0.18 & $0.03-0.89$ & 0.048 & 2.77 & $1.05-7.36$ & 0.040 \\
\hline
\end{tabular}

biopsy (median OS: 171 days after the craniotomy vs 85 days after the biopsy). ${ }^{20}$ Regarding adjuvant treatment modality, there is a significant improvement in mean OS in patients receiving adjuvant chemotherapy after CCRT (Rth 10.9 months vs CCRT only 11.1 months vs CCRT + adjuvant chemotherapy 28.4 months $(P=0.007)$. This is in line with recent data of Perry et al, that states that the addition of chemotherapy concurrent with radiation and after completion of radiation added many benefits to treatment outcomes of elderly patients. His study included 562 patients with GBM aged $\geq 65$ years and compared hypo-Rth ( $40 \mathrm{~Gy} / 15 \mathrm{Fr}$ ) alone vs hypo-Rth with three weeks of concomitant TMZ plus monthly adjuvant TMZ until progression or completion of 12 cycles. Combining TMZ with hypo-Rth was tolerable and resulted in prolonged OS and PFS in all GBM patient groups. Hypo-Rth plus TMZ was superior in median OS and PFS than radiation alone (9.3 and 5.3 months vs 7.6 and 3.9 months, respectively; HR: 0.67 for OS and 0.50 for PFS). No difference was noted in QOL, but patients in the radiotherapy plus TMZ group demonstrated high levels of nausea, vomiting, and constipation. ${ }^{11}$

Studies have shown promising survival with the use of extended temozolomide (E-TMZ) compared to conventional six cycles of temozolomide (C-TMZ) in malignant gliomas; however, the reports are mostly limited to retrospective studies with significant bias. ${ }^{21,22}$

Our study demonstrated that the number of adjuvant chemotherapy cycles had a significant impact on both median PFS and OS. The median PFS in patients receiving $<6$ cycles of chemotherapy was seven months (95\%CI: 5.842-8.158) vs 12 months (95\%CI: $6.979-17.021)$ in patients receiving $\geq 6$ cycles of chemotherapy $(P=0.025)$. Therefore, even in elderly patients extended regimen of temozolomide inferred a positive impact on survival if tolerated.

In the present study the fractionation of radiotherapy showed OS in the conventional radiotherapy group (14 months (95\% CI: 9.787-180,213) vs nine months (95\%CI: 6.009-11.991) in hypofractionation group $(P=0.005)$. In further analysis of patients receiving conventional fractionation, the median OS is much improved in the $<70$ age group ( 15 months vs 11 months, $P=0.176$ ) and $\geq 2$ PS group ( 15 months vs six months, $P=0.629$ ). Roa et al conducted a randomized controlled trial comparing standard radiotherapy ( $60 \mathrm{~Gy} / 30 \mathrm{Fr}$ ) with hypofractionated radiotherapy (40 Gy/15 Fr) for 100 postsurgical patients with GBM aged $\geq 60$ years. OS between conventional Rth and hypofractionated Rth (5.1 and 5.6 months, respectively) was not significantly different, also no difference was noted in Karnofsky performance status (KPS), but steroid use was more frequent in the conventional Rth. ${ }^{8}$ Minniti et al retrospectively studied patients with GBM aged $\geq 65$ years treated with conventional Rth ( $60 \mathrm{~Gy} / 30 \mathrm{Fr}$ ) vs hypofractionated Rth $(40 \mathrm{~Gy} / 15 \mathrm{Fr}$ ) both with concomitant and adjuvant TMZ. Median OS and PFS did not differ between the two treatment arms (12 and 5.6 months for conventional Rth, and 12.5 and 6.7 months for hypofractionated Rth, respectively). However, conventional Rth with TMZ was associated with a significant increase in grade 2 and 3 neurological toxicity, decreased KPS scores, and high steroid requirement. ${ }^{23}$

\section{Conclusion}

Interpretation of the present findings is limited by the retrospective nature of the data. Further research is needed to determine the optimal management of older patients with GBM. The study demonstrated the benefit of adding radiation 
therapy and adjuvant temozolomide for elderly patients with glioblastoma. The ideal radiotherapy fractionation and the number of chemotherapy cycles given in this population remains an ongoing question. Increased availability and utilization of molecular markers such as MGMT methylation status are now helping to select the patients most likely to benefit from temozolomide.

\section{Acknowledgments}

The authors would like to thank the research centre at King Fahad Medical City for supporting this study and to all our radiation oncology staff members.

\section{Disclosure}

The authors report no conflicts of interest in this work.

\section{References}

1. Dolecek TA, Propp JM, Stroup NE, et al. CBTRUS statistical report: primary brain and central nervous system tumors diagnosed in the United States in 2005-2009. Neuro Oncol. 2012;14(Suppl 5):v1-v49. doi:10.1093/neuonc/nos218

2. Chakrabarti I, Cockburn M, Cozen W, et al. A population-based description of glioblastoma multiforme in Los Angeles County, $1974-1999$. Cancer. 2005;104(12):2798-2806. doi:10.1002/cncr.21539

3. Stupp R, Mason WP, van den Bent MJ, et al.; European Organisation for Research and Treatment of Cancer Brain Tumor and Radiotherapy Groups; National Cancer Institute of Canada Clinical Trials Group. Radiotherapy plus concomitant and adjuvant temozolomide for glioblastoma. $N$ Engl J Med. 2005;352(10):987-996. PMID: 15758009. doi:10.1056/NEJMoa043330

4. Iwamoto FM, Reiner AS, Panageas KS, et al. Patterns of care in elderly glioblastoma patients. Ann Neurol. 2008;64(6):628-634. doi:10.1002/ ana. 21521

5. Barnholtz-Sloan JS, Maldonado JL, Williams VL, et al. Racial/ethnic differences in survival among elderly patients with a primary glioblastoma. $J$ Neurooncol. 2007;85(2):171-180. doi:10.1007/s11060-007-9405-4

6. Walker GV, Li J, Mahajan A, et al. Decreasing radiation therapy utilization in adult patients with glioblastoma multiforme. Cancer. 2012;118 (18):4538-4544. doi:10.1002/cncr.27439

7. Scott J, Tsai YY, Chinnaiyan P, et al. Effectiveness of radiotherapy for elderly patients with glioblastoma. Int J Radiat Oncol Biol Phys. 2011;81 (1):206-210. doi:10.1016/j.jijrobp.2010.04.033

8. Keime-Guibert F, Chinot O, Taillandier L, et al. Radiotherapy for glioblastoma in the elderly. $N$ Engl J Med. 2007;356(15):1527-1535. doi:10.1056/NEJMoa065901

9. Roa W, Brasher PM, Bauman G, et al. Abbreviated course of radiation therapy in older patients with glioblastoma multiforme: a prospective randomized clinical trial. J Clin Oncol. 2004;22(9):1583-1588. doi:10.1200/JCO.2004.06.082

10. Malmström A, Grønberg BH, Marosi C, et al.; Nordic Clinical Brain Tumour Study Group (NCBTSG). Temozolomide versus standard 6-week radiotherapy versus hypofractionated radiotherapy in patients older than 60 years with glioblastoma: the Nordic randomised, Phase 3 trial. Lancet Oncol. 2012;13(9):916-926. PMID: 22877848. doi:10.1016/S1470-2045(12)70265-6

11. Wick W, Platten M, Meisner C, et al.; NOA-08 Study Group of Neuro-oncology Working Group (NOA) of German Cancer Society. Temozolomide chemotherapy alone versus radiotherapy alone for malignant astrocytoma in the elderly: the NOA-08 randomised, phase 3 trial. Lancet Oncol. 2012;13(7):707-715. PMID: 22578793. doi:10.1016/S1470-2045(12)70164-X

12. Perry JR, Laperriere N, O'Callaghan CJ, et al. Short-course radiation plus temozolomide in elderly patients with glioblastoma. $N$ Engl $J$ Med. 2017;376:1027-1037. doi:10.1056/NEJMoa1611977

13. Scoccianti S, Magrini SM, Ricardi U, et al. Patterns of care and survival in a retrospective analysis of 1059 patients with glioblastoma multiforme treated between 2002 and 2007: a multicenter study by the Central Nervous System Study Group of Airo (Italian Association of Radiation Oncology). Neurosurgery. 2010;67:446-458. doi:10.1227/01.NEU.0000371990.86656.E8

14. Ewelt C, Goeppert M, Rapp M, et al. Glioblastoma multiforme of the elderly: the prognostic effect of resection on survival. $J$ Neurooncol. 2011;103:611-618. doi:10.1007/s11060-010-0429-9

15. Burton E, Ugiliweneza B, Woo S, Skirboll S, Boaky M, Surveillance A. Epidemiology and end results-medicare data analysis of elderly patients with glioblastoma multiforme: treatment patterns, outcomes and cost. Mol Clin Oncol. 2015;3:971-978. doi:10.3892/mco.2015.590

16. Li SW, Qiu XG, Chen BS, et al. Prognostic factors influencing clinical outcomes of glioblastoma multiforme. Chin Med J (Engl). 2009;122:12451249.

17. Tait MJ, Petrik V, Loosemore A, et al. Survival of patients with glioblastoma multiforme has not improved between 1993 and 2004 : analysis of 625 cases. Br J Neurosurg. 2007;21:496-500. doi:10.1080/02688690701449251

18. Tugcu B, Postalci LS, Gunaldi O, et al. Efficacy of clinical prognostic factors on survival in patients with glioblastoma. Turk Neurosurg. 2010;20:117-125. doi:10.5137/1019-5149.JTN.2461-09.4

19. Cheng H-B, Yue W, Xie C, Zhang R-Y, Hu -S-S, Wang Z. IDH1 mutation is associated with improved overall survival in patients with glioblastoma: a meta-analysis. Tumour Biol. 2013;34(6):3555-3559. doi:10.1007/s13277-013-0934-5

20. Vuorinen V, Hinkka S, Färkkilä M, Jääskeläinen J. debulking or biopsy of malignant glioma in elderly people - a randomised study. Acta Neurochir (Wien). 2003;145:5-10. doi:10.1007/s00701-002-1030-6

21. Seiz M, Krafft U, Freyschlag CF, et al. Long-term adjuvant administration of temozolomide in patients with glioblastoma multiforme: experience of a single institution. J Cancer Res Clin Oncol. 2010;136(11):1691-1695. doi:10.1007/s00432-010-0827-6

22. Malkoun N, Chargari C, Forest F, et al. Prolonged temozolomide for treatment of glioblastoma: preliminary clinical results and prognostic value of p53 overexpression. J Neurooncol. 2012;106(1):127-133. doi:10.1007/s11060-011-0643-0 
23. Minniti G, Scaringi C, Lanzetta G, et al. Standard (60 Gy) or short-course (40 Gy) irradiation plus concomitant and adjuvant temozolomide for elderly patients with glioblastoma: a propensity-matched analysis. Int J Radiat Oncol Biol Phys. 2015;91:109-115. doi:10.1016/j. ijrobp.2014.09.013

\section{Publish your work in this journal}

OncoTargets and Therapy is an international, peer-reviewed, open access journal focusing on the pathological basis of all cancers, potential targets for therapy and treatment protocols employed to improve the management of cancer patients. The journal also focuses on the impact of management programs and new therapeutic agents and protocols on patient perspectives such as quality of life, adherence and satisfaction. The manuscript management system is completely online and includes a very quick and fair peer-review system, which is all easy to use. Visit http://www.dovepress.com/testimonials.php to read real quotes from published authors.

Submit your manuscript here: https://www.dovepress.com/oncotargets-and-therapy-journal 